\title{
Novel composite trachea grafts using 3-dimensional printing
}

\author{
Joanna F. Weber, PhD, ${ }^{\mathrm{a}, \mathrm{b}}$ Sadiq S. Rehmani, MD, ${ }^{\mathrm{c}}$ Mirza Zain Baig, MD, ${ }^{\mathrm{a}, \mathrm{b}}$ Robert Lebovics, MD, ${ }^{\mathrm{d}}$ \\ Wissam Raad, MD, ${ }^{\mathrm{e}}$ Cliff Connery, MD, ${ }^{\mathrm{b}}$ and Faiz Y. Bhora, MD ${ }^{\mathrm{a}, \mathrm{b}}$
}

\section{ABSTRACT}

Objective: Porcine-derived small intestine submucosa (SIS) extracellular matrix (ECM) surgical patches claim to have greater regenerative properties compared with dermal extracellular matrices. We hypothesized that using SIS-ECM in a bioengineered composite tracheal graft would allow better incorporation into the native tissue.

Methods: Two types of size-matched polycaprolactone support scaffolds were designed: rigid and flexible. The SIS-ECM was wrapped around the polycaprolactone supports lining the inside and outside of the graft. The grafts were implanted in 4 Yorkshire pigs, replacing an $\sim 2 \mathrm{~cm}$ segment of native trachea. Airway patency was evaluated with computed tomography scans and explanted grafts were examined grossly and histologically.

Results: All animals survived through the immediate postoperative period. Generally, extraluminal examination showed a smooth transition between native and graft without significant volumetric loss. Animals that received the flexible design survived $\sim 10$ days longer than those that received the rigid design; however, severe perianastomotic intraluminal granulation tissue was observed. The rigid design had less significant intraluminal granulation tissue development at the distal anastomosis, but partial dehiscence had occurred at the proximal anastomosis interrupting graft incorporation.

Conclusions: The generally good extraluminal graft incorporation in our composite tracheal graft highlights some increased regenerative capabilities of SIS-ECM. However, the presence of intraluminal granulation tissue indicates that its use as an offthe-shelf, unaltered substrate in an airway graft is still not ideal. Further research must be conducted to determine whether a modification of the substrate is possible to enhance luminal airway incorporation and to exert control over the mechanisms responsible for granulation tissue development. (JTCVS Open 2021;5:152-60)

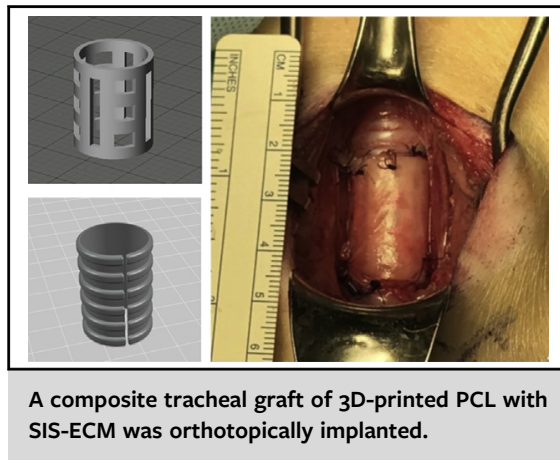

CENTRAL MESSAGE

SIS-ECM has some increased

regenerative capabilities over

dermal ECM; however, its off-theshelf use as an airway graft lacks

appropriate properties for

luminal integration and

epithelialization.

\section{PERSPECTIVE}

The findings of this study highlight the importance of rigorous testing of surgical patch materials in airway reconstruction before use in human patients. Although these second-generation ECMs promise increased regenerative potential, more studies both to further increase regenerative effects and decrease intraluminal granulation are needed before wider clinical adoption.

See Commentary on page 161.

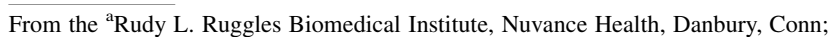
${ }^{b}$ Department of Thoracic Surgery, Nuvance Health, Poughkeepsie, NY; Departments of ${ }^{\mathrm{c}}$ Internal Medicine and ${ }^{\mathrm{e}}$ Thoracic Surgery, Icahn School of Medicine at Mount Sinai, and ${ }^{\mathrm{d}}$ Department of Otolaryngology, Mount Sinai West, Mount Sinai Health System, New York, NY.

Supported by The Hussain Family Foundation.

Received for publication Oct 30, 2020; accepted for publication Nov 5, 2020; available ahead of print Dec 8, 2020.

Address for reprints: Faiz Y. Bhora, MD, Rudy L. Ruggles Biomedical Institute, 131 West St, Danbury, CT 06810 (E-mail: faiz.bhora@nuvancehealth.org).

2666-2736

Copyright $\odot 2020$ The Authors. Published by Elsevier Inc. on behalf of The American Association for Thoracic Surgery. This is an open access article under the CC BY-NC-

ND license (http://creativecommons.org/licenses/by-nc-nd/4.0/).

https://doi.org/10.1016/j.xjon.2020.11.001
The morbidity and mortality due to chronic airway stenosis is significant. ${ }^{1}$ Tracheal stenosis is caused by prolonged intubation, tracheostomy complications, cancer, infection, inflammatory conditions, and congenital malformations. ${ }^{2-4}$ It has been estimated that up to $10 \%$ of patients will develop postintubation tracheal stenosis. ${ }^{5}$ Of these cases, $1 \%-2 \%$ are symptomatic or severe, but it is difficult to estimate how many involve long segments of the trachea. ${ }^{6}$ Reconstructing a long segment of the trachea poses a unique surgical problem because often it is not possible to simply resect a significant length of the trachea and be left with enough length for a primary end to end anastomosis. Surgeons have utilized decellularized extracellular matrix 

Abbreviations and Acronyms
3D $=3$-dimensional
$\mathrm{CT}=$ computed tomography
$\mathrm{ECM}=$ extracellular matrix
$\mathrm{PCL}=$ polycaprolactone
SIS $=$ small intestine submucosa

(ECM) materials to make up for the needed length in rare cases, ${ }^{7}$ and this is a logical move because these materials are already successfully used in many other surgical grafting applications in place of synthetic materials or autologous tissue transplants.

In general, ECMs make excellent replacements for tissue reconstruction since they are derived from native tissues. Although processed to remove cells, the structural integrity of the tissue remains and therefore it contains components readily found in native tissues. These physiochemical properties of ECMs can help to accelerate integration of the graft and healing of the wound. ${ }^{8,9}$ The process of decellularization removes the cellular components from the source tissues, greatly reducing the potential of immunogenic reactions. ${ }^{10}$ Because these decellularized ECMs are largely composed of highly conserved dense structural proteins such as collagen, they are both shelf-stable and crossspecies compatible, further reducing the immunogenic complications and the logistics associated with using fresh human donor-derived tissue.

A second-generation of decellularized ECM patches are derived from porcine small intestine submucosa (SIS). These are processed using an improved decellularization process that further reduces cell remnants such as nuclear and cell membrane fragments that can stimulate an apoptotic response in the surrounding native tissue. ${ }^{11}$ The SIS-ECM patches are not treated with a crosslinking glutaraldehyde solution, leaving native binding sites intact and available for interaction with the recipient's cells, theoretically increasing the regenerative capacity of these ECMs and minimizing possible inflammatory reactions. ${ }^{12}$ SIS-ECMs are also thinner than dermal ECMs, allowing for more rapid remodeling of the patch.

Successfully using ECMs in tracheal reconstruction requires careful consideration of the structural elements of the native trachea. The trachea is a long flexible fibrous tube made mostly of dense but elastic connective tissues which easily accommodates changes in length and flexion associated with normal physiological movements. Cartilage rings located at regular intervals along the length of the trachea provide radial rigidity that maintain airway patency and prevent malacia and collapse. Although dermal ECMs can provide some rigidity due to mechanical properties inherent to their thickness, preventing malacia when used in a tracheal graft necessitates the use of an additional support structure. ${ }^{13}$

Previously, our group has used polycaprolactone (PCL)supported ECMs to repair partial tracheal defects in a large animal model. ${ }^{13,14}$ In our previous studies, our PCL scaffolds produced on a 3-dimensional (3D) printer were successful in mitigating graft malacia and maintained an open airway throughout study duration. In some instances, it appeared that the bovine dermal ECM caused extensive perianastomotic granulation tissue, resulting in obstructive stenosis. ${ }^{14}$

Therefore, in this study we sought to build on our previous work and explored the feasibility of using SISECMs in a composite PCL-ECM graft to replace a circumferential section of the trachea in the large animal model. The porcine-derived SIS-ECM is expected to have fewer immunogenic concerns when used in the pig implant model due to matching donor and recipient species and the increased regenerative properties of this secondgeneration ECM type are hypothesized to reduce or eliminate granulation tissue development. In addition, we also hypothesize that the SIS-ECM will provide a better substrate for airway epithelial migration because of the similarity in cell type to the mucous-producing epithelial layer found in native SIS.

\section{MATERIALS AND METHODS}

Feasibility of using SIS-ECM in a trachea graft was assessed with a small group of animals. All animal work was carried out adhering to animal use guidelines set by the Mount Sinai Institutional Animal Care and Use Committee. Four female Yorkshire pigs (approximate age 2 months, approximate weight $30 \mathrm{~kg}$ ) were purchased from Animal Biotech Industries (Doylestown, Pa). Animals were acclimated to the facility for several days before any procedures were started.

\section{Graft Design and Preparation}

To ensure size-matched grafts, animals underwent pre-operative computed tomography (CT) scans. Trachea lumen diameter and trachea wall thickness were measured from several CT scan slices and averaged. Two types of PCL supports (rigid $[\mathrm{n}=2]$ or flexible $[\mathrm{n}=2]$ ) were designed using the computer-aided drawing program Autodesk Fusion 360 (Autodesk, San Rafael, Calif) to match the average size of the tracheas (Figure 1). The drawings were exported to the 3D printer as stereolithography files, a standard file format widely used in computer-aided manufacturing including 3D printing and rapid prototyping. The PCL supports were printed by melt extrusion on a MakerBot Replicator 2 desktop 3D printer (MakerBot Industries, New York, NY) and sanitized overnight by submersion in hydrogen peroxide followed by 1 hour of ultraviolet light exposure in a laminar flow hood. A section of a SIS-ECM sheet (Cook Biotech, West Lafayette, Ind) was cut based on the dimensions of the PCL support such that a single sheet could be used to create nested cylinders that would wrap around the PCL support, lining the inside and outside of the graft. Excess material was trimmed, and the seams of the ECM were approximated and secured using interrupted sutures. Printing of each support structure took less than an hour and the overall time for graft preparation, including printing, sterilizing, and attaching ECM was approximately 24 hours. The resulting composite grafts were rehydrated in sterile saline before implantation. 


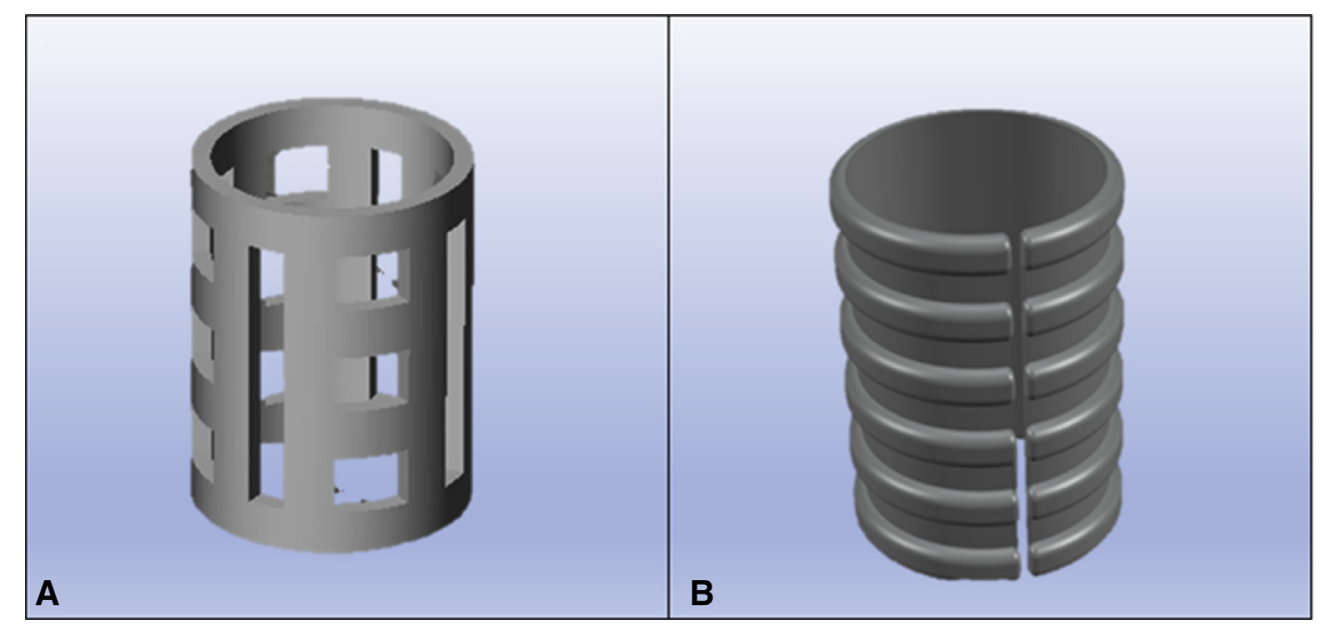

FIGURE 1. Three-dimensional (3D) rendering of polycaprolactone printed grafts for (A) the rigid scaffold and (B) the flexible scaffold. The grafts were designed in Autodesk Fusion 360. After scanning the animals, the diameters of the grafts were size-matched to the trachea of each animal. The grafts were then 3D printed by fused deposition using a MakerBot benchtop printer.

\section{Surgical Approach}

Implantation followed previously reported procedures (Figure 2). Sedated and intubated animals were place in a supine position on the operating table. The trachea was accessed via midline cervical incision and strap muscle separation. Dissection of remaining surrounding structures further exposed the trachea. For each animal, a 4-ring circumferential tracheal segment was removed and replaced with the graft. Cross-table ventilation was used while the proximal anastomosis to the graft was completed. After securing the proximal anastomosis, the endotracheal tube was re-advanced through the graft and into the distal trachea. The distal anastomosis was completed around the endotracheal tube. Integrity of the anastomosis was assessed by flooding the surgical site with sterile saline and ventilating to $20-30 \mathrm{~cm} \mathrm{H}_{2} \mathrm{O}$ of pressure. Strap muscles were re-approximated, a Penrose drain was placed, and the incision was closed in layers. Prophylactic antibiotic ointment was applied to the incision.

\section{Postoperative Care}

Animals stayed in the operating room while sedation was reversed and for extubation. After extubation, animals were moved to individual recovery pens where breathing and oxygen saturation were closely monitored. Once fully awake and stable, animals were given food and water. On postoperative day 1 , animals were moved to standard housing. On postoperative day 2, the drain was removed. Animals were examined daily by the veterinary staff and closely monitored for signs of respiratory distress and infection. Postoperative pain medication, dexamethasone, and guaifenesin were administered by the veterinary staff. CT scans were scheduled for every 2 weeks postoperation.

\section{Sample Preparation}

Following death, a full necropsy on each animal was performed by a member of the veterinary staff. The graft, trachea, and surrounding structures were visualized in situ before removal and gross examination. Samples were then fixed in formalin, paraffin embedded, and sectioned. Sections from the trachea and graft were cut such that the transition between native tissue and graft could be examined. Sections were stained with hematoxylin and eosin for overall morphology.

\section{RESULTS \\ Clinical Outcomes}

All animals remained clinically stable during the immediate postoperative period and up until postoperative day 12 , showing no signs of infection or respiratory distress.
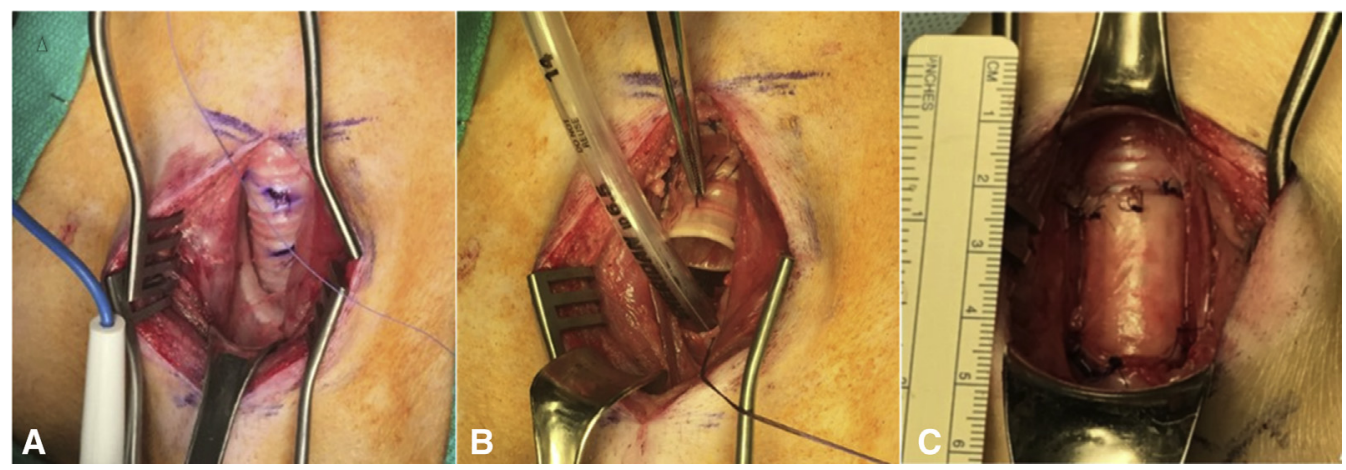

FIGURE 2. Surgical approach for orthotopic transplantation of the tracheal graft follows our previous reported procedure. A, The trachea was exposed with a midline incision and strap muscles were separated. Approximately $2 \mathrm{~cm}$ of the native trachea was marked for removal. B, The proximal anastomosis was secured, whereas cross-table ventilation was provided. C, The distal anastomosis was secured after the endotracheal tube was re-advanced through the graft. 
On postoperative day 13, 1 animal from the rigid scaffold group was found dead. A CT scan of the other animal in this group showed a patent graft with no stenosis, but indications of partial dehiscence at the proximal anastomosis. Although this animal was still clinically stable, showing no signs of distress, the consensus after consultation with the veterinary team was to electively put to death the animal to avoid sudden death. CT scans at postoperative day 13 of the animals with the flexible graft showed patent grafts, but significant intraluminal granulation tissue at both proximal and distal anastomoses, which was partially occluding the airway. Stenting was done in an animal with $>50 \%$ occlusion to maintain airway patency. At postoperative day 21, the animal that received the stenting began to display severe signs of respiratory distress and was humanely killed. CT scanning on postoperative day 24 of the remaining animal showed $>50 \%$ occlusion, thus stenting was planned. Although the animal appeared clinically stable with barely detectable stridor, the animal was found dead on postoperative day 26 , before the stenting procedure could be performed.

\section{Gross Examination}

From the outside, the tracheas of all animals appeared intact with well-healed distal anastomoses and seamless transition without significant size differences between graft and native trachea. Well-healed proximal anastomoses were observed in the flexible graft group, whereas partial dehiscence $(\sim 50 \%)$ at the proximal anastomosis was observed in both animals in the rigid graft group. In 1 rigid graft animal, there was fistulous communication between the trachea and esophagus at the partial dehiscence. For all animals, the PCL scaffold was still firmly seated and intact and neither support type showed evidence of malacia or midgraft stenosis. Significant peri-anastomotic intraluminal granulation tissue was observed in the flexible graft group, whereas the rigid graft group showed less significant intraluminal granulation tissue at the dehiscence and even a smooth transition between graft and native at the distal anastomosis (Figure 3).

\section{Histologic Examination}

Extraluminal granulation tissue was organized, densely collagenous, and mildly inflamed. There was an abundance of inflammatory cells on a background of fibroplasia and a maturing capillary bed. Examination of both animals that received the rigid grafts showed extensive infection with intralesional bacteria at the site of dehiscence at the proximal anastomosis with loosely arranged plump spindle cells infiltrated by lymphocytes, neutrophils, and macrophages. Similar findings but with significantly less granulation tissue were observed at the site of distal anastomosis. In both animals that received the flexible grafts, the significant luminal granulation tissue at both the proximal and distal anastomoses contained loosely arranged plump spindle cells infiltrated by macrophages in well-vascularized, but immature tissue. In all animals, regardless of graft type, mineralization of the luminal ECM layer was observed as a homogenous to granular brittle material (Figure 4).

\section{DISCUSSION}

Long-segment tracheal repair or replacement necessitates the use of grafting material due to inherent anatomical limitations. Donor transplant material has not been successful and other autologous techniques for tracheal replacement have had limited success. ${ }^{15-17}$ As such, surgeons have looked to using off-the-shelf synthetic and natural surgical grafting materials. ${ }^{18,19}$ In this study, summarized in Figure 5, we examined the feasibility of using a composite tracheal graft composed of a 3D-printed PCL scaffold supporting a porcine-derived SIS-ECM. These grafts were implanted orthotopically in a pig model to assess their mechanical and regenerative functionality. Table 1 highlights differences in the graft design attributes used in this study compared with our pervious study using dermal ECM. ${ }^{14}$ The large animal model is necessary to create a clinically relevant model to human anatomy. ${ }^{20,21}$ Our work is also novel in that we are testing our bioengineered grafts in an orthotopic transplantation model.

Although a high number of sudden deaths and sudden onset of clinical symptoms could be considered a detractor of this model, we believe that they can be a means to an accelerated graft evaluation provided that early observations are noted. Future implantation work will closely monitor the animals during the immediate postoperative period with more frequent $\mathrm{CT}$ scanning to better capture any changes as they occur.

The PCL supports were easily customized and quickly manufactured with a benchtop 3D printer without the need for postprinting processing or surface treatments to remove residual monomers. Benchtop 3D printers themselves are financially accessible, with simple single-nozzle models costing around $\$ 2000$. Print feedstock materials are also quite accessible, many costing well under $\$ 1$ per gram. Many benchtop fused filament 3D printers are capable of using a variety of plastic polymers as feedstock and hobbyists most commonly use polylactic acid and acrylonitrile butadiene styrene because their high melting point and stiffness result in 3D printed parts that hold their shape well under a variety of conditions. PCL is a less common feedstock in hobby $3 \mathrm{D}$ printing due to its relatively low $\left(\sim 60^{\circ} \mathrm{C}\right)$ melting point and lower strength that gives its printed products more flexibility. PCL is biocompatible and biodegradable and is still a great material for fused filament fabrication in 3D printers. PCL has been shown to be biocompatible and has been Food and Drug Administration approved for implantation in the human body. ${ }^{22}$ Under physiological conditions, PCL is degraded by hydrolysis 


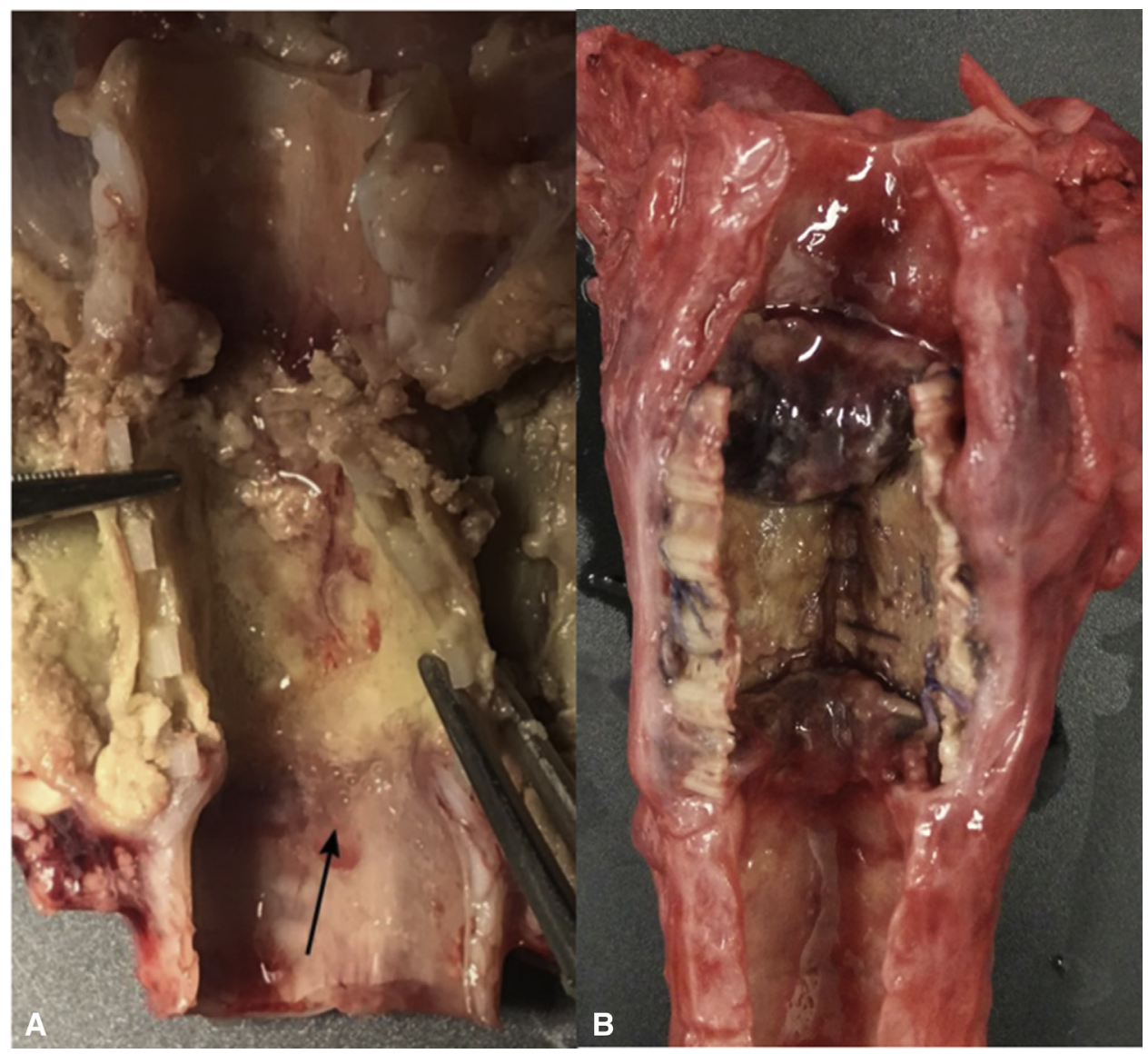

FIGURE 3. Upon gross examination of the explanted trachea grafts at necropsy, intraluminal incorporation into the native tissue was extremely variable, although both graft designs were made from the same materials. The rigid scaffolds (A) appeared to have a smooth transition between the graft and native tissue at the distal anastomosis (arrow), whereas the proximal anastomosis showed dehiscence. On the other hand, the flexible scaffolds (B) showed significant intraluminal granulation tissue development protruding into the airway at both proximal and distal anastomoses that substantially occluded the airway. Variability in the anastomotic healing between the 2 graft design types indicates that scaffold rigidity is an important factor in successful trachea graft designs.

in a relatively slow and predictable timeline, without producing toxic degradation products. ${ }^{23,24}$ These properties make it a useful material to use as temporary support for maintaining mechanical strength to support a patent tracheal lumen while the body has a chance to grow new cartilage.

Table 2 summarizes the outcome measures for this study and includes a comparison to our previous study. The design of the graft appeared to be an important factor in successfully accommodating the longitudinal traction and shear forces at the anastomoses. The flexible graft was apparently able to minimize forces both at the proximal and distal anastomoses, whereas the rigid graft was unable to accommodate forces at the proximal anastomosis resulting in dehiscence. In the pig model, we are unable to immobilize neck movement as would be done with human patients; thus, the normal range of motion is resumed as soon as sedation is reversed, immediately subjecting the graft and anastomoses to mechanical stresses. In humans, the normal range of neck motion between flexion and extension can cause the trachea to stretch almost $2 \mathrm{~cm}$. Much of this change occurs in the section of trachea between the vocal folds and the sternal notch and we expect a similar degree of movement to occur in pigs. ${ }^{25,26}$ Although we expect the principal force to be tensional, some shear forces are also developed due to the bending of the trachea. The native trachea of both humans and pigs is extremely elastic and pliable in the longitudinal direction, allowing for this motion. Thus, it is unsurprising that the flexible design of the graft, which more closely mimics the longitudinal extensibility of the native trachea, was better able to accommodate this change in length without resulting in dehiscence.

The extraluminal incorporation of the graft highlighted the improved regeneration potential of the SIS-ECM with a smooth transition from native tissue to graft without significant volumetric loss, less hemorrhagic granulation tissue, and reduced inflammation indicative of wound resolution (Figure 4, D). However, the success of the flexible 


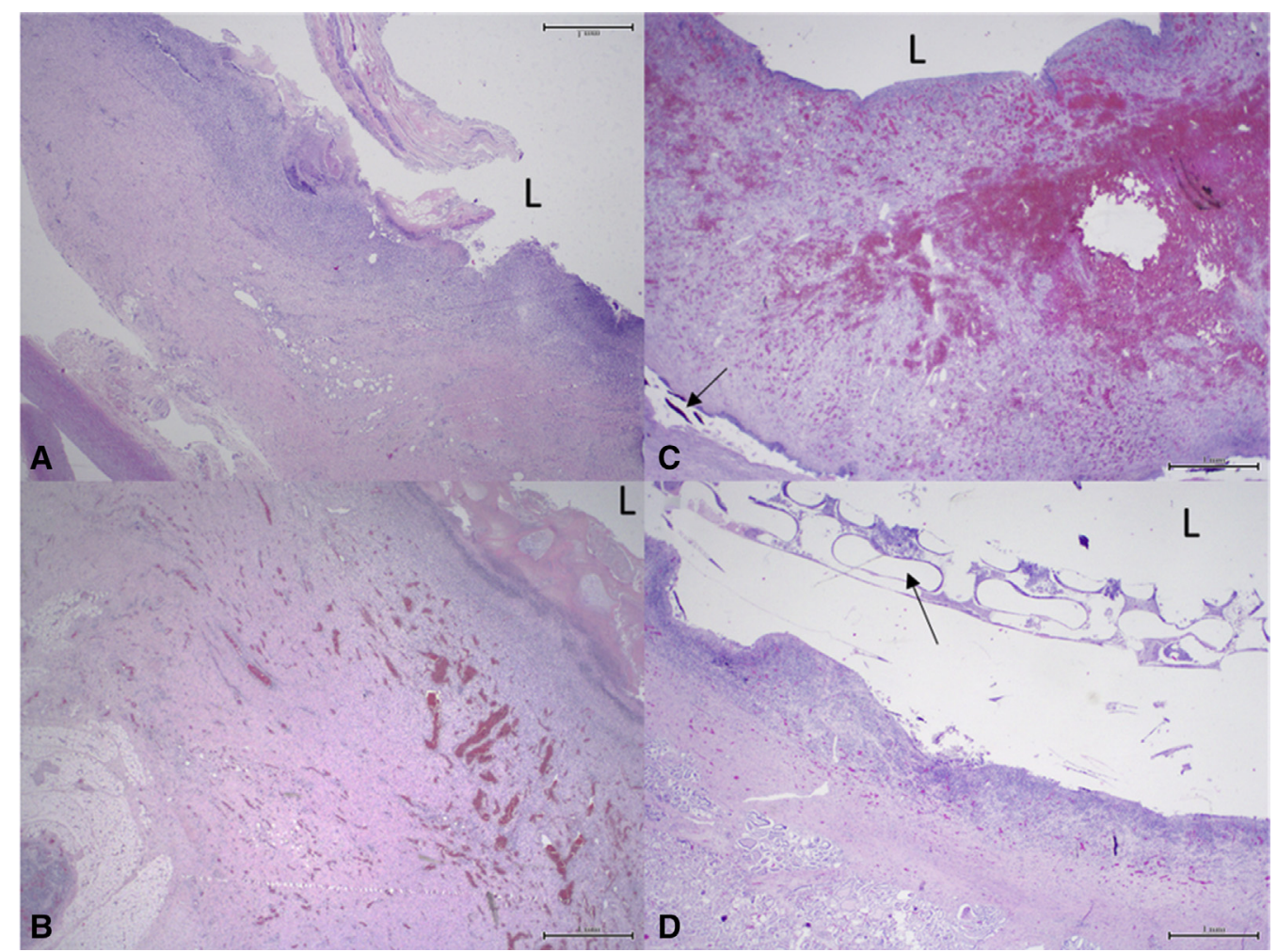

FIGURE 4. Representative hematoxylin and eosin histological images of the anastomotic interface between graft and native trachea of the rigid scaffold graft (A and B) and the flexible scaffold graft (C and D). A, The distal anastomosis shows mild granulation tissue suggestive of progress toward good wound resolution and graft incorporation. B, Closer to the proximal anastomosis, where the dehiscence occurred, extraluminal granulation tissue was more hemorrhagic and infectious material was observed in the lumen. C, The hemorrhagic granulation tissue protruded into the lumen; the arrow indicates a calcified remnant of the small intestine submucosa extracellular matrix. D, Extraluminal granulation tissue is significantly less inflamed and less hemorrhagic, suggesting that the exterior of the graft was becoming well incorporated; the arrow indicates void spaces where the polycaprolactone scaffold was located. Functional airway epithelium was not observed in any of the grafts likely because both granulation tissue and anastomotic dehiscence can significantly impede the migration of native epithelium. $L$, Lumen. Scale bar $=1 \mathrm{~mm}$.

graft was significantly hindered by the formation of intraluminal granulation tissue, evident upon gross examination of the trachea lumen, at both the proximal and distal anastomoses. In the rigid graft, intraluminal granulation tissue was only evident upon close examination in histologic sections. Differences in the growth of granulation tissue between the 2 groups of animals may be in part explained by the longer survival time of 1 group over the other; however, additional factors such as anastomotic tension or a foreign body reaction could also be at play. Because the granulation tissue was seen as early as 13 days postoperatively in the flexible group, we hypothesize that mechanical stress at the anastomosis is a major contributor. The observation of less-extensive granulation tissue at the distal end of the rigid grafts suggests that tension at that distal anastomosis was reduced by the proximal dehiscence and supports a mechanical cause for the granulation tissue formation. ${ }^{27}$

Granulation tissue formation is a significant problem in surgical interventions of the airway. Whereas granulation tissue is part of the normal wound healing process, persistent granulation tissue and/or significantly hypertrophic intraluminal granulation tissue can obstruct the airway, leading to sudden mortality. The cause of airway granulation formation is not completely understood, but several mechanisms have been proposed, including a systemic immune response mediated by B- and T-cells, a local tissue response mediated by abnormal fibroblasts, and a hypoxiamediated hypertrophic angiogenic response. ${ }^{28-30}$ In this study, dexamethasone was given to the animals for several days postoperatively in an effort to reduce granulation tissue formation. Due to the untargeted nature of systemic steroids, this treatment is not ideal because it can have off-target effects such as delayed healing and increased chance of infection.

Based on the results of this study, it appears that using SIS-ECM in place of dermal ECM provides no measurable advantages with regard to intraluminal incorporation. In addition, commercial SIS-ECM is manufactured by combining up to 4 layers of tissue together to increase strength, but it is still significantly thinner than dermal 


\section{Testing Novel Composite 3D-Printed Trachea Grafts in a Large Animal Model}

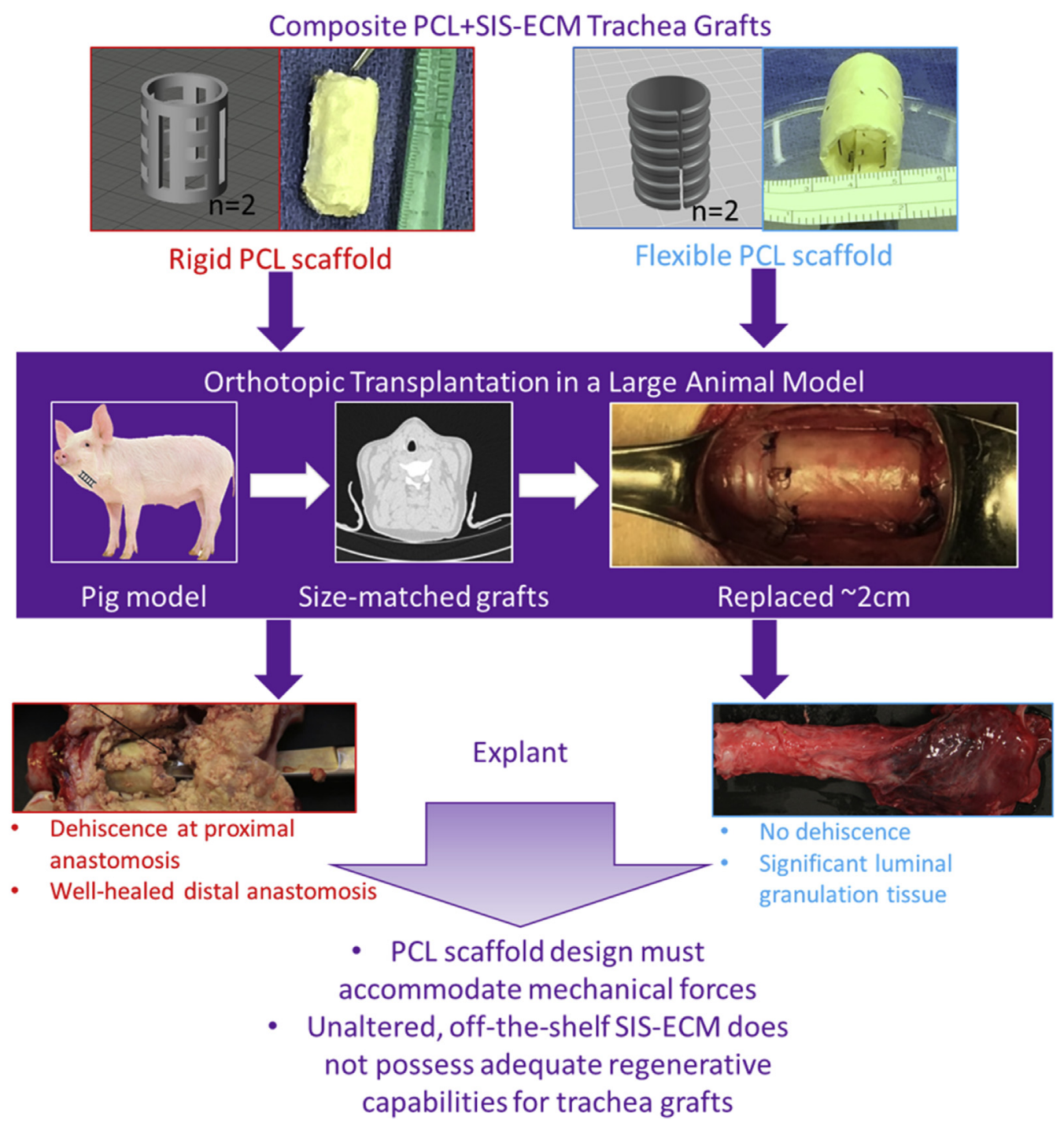

FIGURE 5. Orthotopic transplantation of a composite trachea graft was studied in a large animal model. Size-matched scaffolds were 3-dimensional (3D)printed using polycaprolactone $(P C L)$ by measuring trachea dimensions from preoperative scans. The PCL scaffolds $(\mathrm{flexible}[\mathrm{n}=2]$ and rigid $[\mathrm{n}=2])$ were wrapped intra- and extraluminally with extracellular matrix $(E C M)$ patches derived from porcine small intestine submucosa (SIS). The rigid PCL scaffold was unsuccessful at accommodating tension and shear forces, resulting in dehiscence at the proximal anastomosis. The flexible graft was able to accommodate these forces without dehiscence, but suffered from extensive intraluminal granulation tissue development. Although SIS-ECM is a secondgeneration ECM patch, this study was unable to observe any increased regenerative capabilities of the ECMs as-is compared with our previous experienced with dermal ECM.

TABLE 1. Summary of graft design attributes in this study compared with the previous study. ${ }^{14}$ Extracellular matrix (ECM) types included dermal and small intestine submucosa (SIS)

\begin{tabular}{lccc}
\hline \multicolumn{1}{c}{ Attribute } & \multicolumn{2}{c}{ This study } & Previous study $^{\mathbf{1 4}}$ \\
\hline Coverage & $360^{\circ}$ & $360^{\circ}$ & $360^{\circ}$ \\
Graft length $(\mathrm{cm})$ & 2 & 2 & 2 \\
Scaffold design & Rigid & Flexible & Rigid \\
ECM type & SIS & SIS & Dermal \\
ECM location & $\begin{array}{c}\text { Intra- and } \\
\text { extraluminal }\end{array}$ & $\begin{array}{c}\text { Intra- and } \\
\text { extraluminal }\end{array}$ & $\begin{array}{c}\text { Intraluminal } \\
\text { only }\end{array}$ \\
\hline
\end{tabular}

ECM. Our design of enclosing the PCL supports in the SISECM to further increase graft strength and ensure an airtight graft, created a dead space between the outer and inner ECM layers. The lack of direct contact of the inner ECM layer to native tissue could have contributed to a delayed integration into the surrounding tissue. This may have contributed to our observation of calcification of the inner ECM layers, although other literature concerning SISECM show that it is more resistant to calcification than other ECM types. ${ }^{31-34}$ Calcification of the inner ECM layer may have also contributed to granulation tissue 
TABLE 2. Outcome summary for all animals. In this study, 2 animals received the rigid scaffold design and 2 animals received the flexible scaffold design, both using small intestine submucosa extracellular matrix (ECM). Two animals from the previous study received a rigid scaffold design with dermal ECM ${ }^{14}$

\begin{tabular}{|c|c|c|c|c|}
\hline Study & Scaffold design & Survival days & Extraluminal observations & $\begin{array}{l}\text { Intraluminal } \\
\text { observations }\end{array}$ \\
\hline \multirow[t]{2}{*}{ This study } & Rigid (n = 2) & $13.5(13-14)$ & $\begin{array}{l}\text { Smooth transition from graft to native at distal anastomosis } \\
\text { Fistula }(\mathrm{n}=1)\end{array}$ & $\begin{array}{l}\text { Mild granulation } \\
\text { ECM calcification }\end{array}$ \\
\hline & Flexible $(\mathrm{n}=2)$ & $23.5(21-26)$ & $\begin{array}{l}\text { Smooth transition between graft and native at both anastomoses } \\
\text { Mild granulation }\end{array}$ & $\begin{array}{l}\text { Severe granulation } \\
\text { ECM calcification }\end{array}$ \\
\hline $\begin{array}{c}\text { Previous } \\
\text { study }\end{array}$ & $\operatorname{Rigid}(\mathrm{n}=2)$ & $29.0(24-34)$ & $\begin{array}{l}\text { Well-incorporated with native tissue at both anastomoses } \\
\text { Mild granulation } \\
\text { Necrotic foci }\end{array}$ & Severe granulation \\
\hline
\end{tabular}

Values for survival days are presented as mean (range).

development by increasing the mechanical stiffness of the graft interior.

The SIS-ECM used in this study was chosen due to its ease of accessibility because it is a commercially available product. In addition, the decellularization process for these and other commercial ECMs are presumed to be very carefully standardized to ensure batch-to-batch consistency. Physiochemical properties of ECMs (including, but not limited to degree of cross-linking, amount of DNA and cellular debris, collagen structure and content, and structure and content of noncollagenous proteins) can vary due to the specific process used and will be different depending on the manufacturer or laboratory. Quantification of these physiochemical properties in future studies of other ECM types may provide valuable data. Unfortunately, the successful use of ECMs in certain applications does not guarantee its success in other applications. Although we expected that this new generation of ECM would perform much better than the dermal ECMs in our graft, we did not find that to be the case.

Relying on cues innate to the ECM substrates to enhance luminal epithelial migration and integration into native tissue is not adequate for airway tissue engineering. The airway is a harsh environment, exposed to constant mechanical stress from movement as well as microbial stresses from contact with the outside environment. Mucus clearance is difficult because the grafted length does not contain ciliated cells that would move the mucus out of the airway. This, in conjunction with the presence of surface irregularities at the anastomoses can contribute to increased collection of infectious material at the graft-native interface. All these factors may result in persistent and hypertrophic granulation tissue, which in turn delays epithelialization and healing of the anastomoses. Further work must focus on modifying the grafting materials to include stronger cues for implant integration so that these obstacles can be overcome. These cues can be translated from other tissue engineered strategies such as incorporating cells that can mediate the immune response and generate proregenerative paracrine signaling molecules that attract surrounding cells to the implanted grafts to speed up integration into the recipient's native tissue. Direct incorporation of chemotactic factors may also be used and would help to avoid the complexities associated with donor- or patient-derived cells.

Previously, we have had longer survival time with partial circumference grafts. Several factors may have contributed to this success, including a relatively smaller graft surface area as well as maintaining some continuity of the native tissue in the uninjured intact tissue opposite the partial graft. Based on our feasibility study here and our previous studies, it is difficult to definitively choose the best design for a full circumferential graft, but using mechanistic studies to elucidate the mechanisms driving trachea wound healing and graft incorporation will help to refine the design both in structure and materials.

\section{CONCLUSIONS}

SIS-ECM combined with a 3D-printed PCL scaffold with a flexible design provided adequate structural integrity to a circumferential tracheal graft to prevent graft malacia and was well-incorporated externally with the native trachea. There was a significant intraluminal response to the matrix that precludes its use as an immediate airway replacement solution in its unaltered, off-the-shelf state. Incorporation of biological factors or cells into these scaffolds and ECMs can potentially decrease the granulation tissue formation and enhance tissue regeneration. Due to the lack of information on tracheal healing and granulation tissue formation, further studies are needed to determine mechanisms responsible for granulation tissue development and to systematically assess the properties of and recipient's response to various acellular scaffolds before consideration of wider, clinical adoption for tracheal reconstruction. 


\section{Conflict of Interest Statement}

The authors reported no conflicts of interest.

The Journal policy requires editors and reviewers to disclose conflicts of interest and to decline handling or reviewing manuscripts for which they may have a conflict of interest. The editors and reviewers of this article have no conflicts of interest.

\section{References}

1. Cotton R. Management of subglottic stenosis in infancy and childhood. Ann Otol Rhinol Laryngol. 1978;87:649-57.

2. Crowley C, Birchall M, Seifalian AM. Trachea transplantation: from laboratory to patient. J Tissue Eng Regen Med. 2015;9:357-67.

3. Lange P, Fishman JM, Elliott MJ, De Coppi P, Birchall MA. What can regenerative medicine offer for infants with laryngotracheal agenesis? Otolaryngol Head Neck Surg. 2011;145:544-50.

4. Napieralska A, Miszczyk L, Blamek S. Tracheal cancer-treatment results, prognostic factors and incidence of other neoplasms. Radiol Oncol. 2016;50:409-17.

5. Kastanos N, Estopá Miró R, Marín Perez A, Xaubet Mir A, Agustí-Vidal A. Laryngotracheal injury due to endotracheal intubation: incidence, evolution, and predisposing factors. A prospective long-term study. Crit Care Med. 1983;11: 362-7.

6. Zias N, Chroneou A, Tabba MK, Gonzalez AV, Gray AW, Lamb CR, et al. Posttracheostomy and postintubation tracheal stenosis: report of 31 cases and review of the literature. BMC Pulm Med. 2008;8:1-9.

7. Haykal S, Salna M, Waddell TK, Hofer SO. Advances in tracheal reconstruction. Plast Reconstr Surg. 2014;2:e178.

8. Piccoli M, Urbani L, Alvarez-Fallas ME, Franzin C, Dedja A, Bertin E, et al. Improvement of diaphragmatic performance through orthotopic application of decellularized extracellular matrix patch. Biomaterials. 2016;74:245-55.

9. Scholl FG, Boucek MM, Chan K-C, Valdes-Cruz L, Perryman R. Preliminary experience with cardiac reconstruction using decellularized porcine extracellular matrix scaffold. World J Pediatr Congenit Heart Surg. 2010;1:132-6.

10. Gilbert TW, Freund JM, Badylak SF. Quantification of DNA in biologic scaffold materials. J Surg Res. 2009;152:135-9.

11. Morris AH, Chang J, Kyriakides TR. Inadequate processing of decellularized dermal matrix reduces cell viability in vitro and increases apoptosis and acute inflammation in vivo. Biores Open Access. 2016;5:177-87.

12. Cramer MC, Badylak SF. Extracellular matrix-based biomaterials and their influence upon cell behavior. Ann Biomed Eng. 2020;48:2132-53.

13. Rehmani SS, Al-Ayoubi AM, Ayub A, Barsky M, Lewis E, Flores R, et al. Threedimensional-printed bioengineered tracheal grafts: preclinical results and potential for human use. Ann Thorac Surg. 2017;104:998-1004.

14. Bhora FY, Lewis EE, Rehmani SS, Ayub A, Raad W, Al-Ayoubi A, et al. Circumferential three-dimensional-printed tracheal grafts: research model feasibility and early results. Ann Thorac Surg. 2017;104:958-63.

15. Grillo HC. Tracheal replacement: a critical review. Ann Thorac Surg. 2002;73: 1995-2004.

16. Osada H. Artificial trachea. J Bronchol. 2006;13:39-43.

17. Fabre D, Kolb F, Fadel E, Mercier O, Mussot S, Le Chevalier T, et al. Successful tracheal replacement in humans using autologous tissues: an 8-year experience. Ann Thorac Surg. 2013;96:1146-55.
18. Wurtz A, Porte H, Conti M, Desbordes J, Copin MC, Azorin J, et al. Tracheal replacement with aortic allografts. $N$ Engl J Med. 2006;355:1938-40.

19. Martinod E, Chouahnia K, Radu DM, Joudiou P, Uzunhan Y, Bensidhoum M, et al. Feasibility of bioengineered tracheal and bronchial reconstruction using stented aortic matrices. JAMA. 2018;319:2212-22.

20. Yahaya B. Understanding cellular mechanisms underlying airway epithelial repair: selecting the most appropriate animal models. Sci World J. 2012;2012: $1-12$.

21. Goh CSL, Joethy JV, Tan BK, Wong M. Large animal models for long-segment tracheal reconstruction: a systematic review. J Surg Res. 2018;231:140-53.

22. Moers-Carpi MM, Sherwood S. Polycaprolactone for the correction of nasolabial folds: a 24-month, prospective, randomized, controlled clinical trial. Dermatol Surg. 2013;39:457-63.

23. Sun H, Mei L, Song C, Cui X, Wang P. The in vivo degradation, absorption and excretion of PCL-based implant. Biomaterials. 2006;27:1735-40.

24. Yang L, Li J, Jin Y, Li M, Gu Z. In vitro enzymatic degradation of the cross-linked poly( $\varepsilon$-caprolactone) implants. Polym Degrad Stab. 2015;112:10-9.

25. Wong DT, Weng H, Lam E, Song HB, Liu J. Lengthening of the trachea during neck extension: which part of the trachea is stretched? Anesth Analg. 2008;107: 989-93.

26. Harris RS. The effect of extension of the head and neck upon the infrahyoid respiratory passage and the supraclavicular portion of the human trachea. Thorax. 1959; 14:176-80.

27. Grinnell F, Zhu M, Carlson MA, Abrams JM. Release of mechanical tension triggers apoptosis of human fibroblasts in a model of regressing granulation tissue. Exp Cell Res. 1999;248:608-19.

28. Lappin DF, Macleod CP, Kerr A, Mitchell T, Kinane DF. Anti-inflammatory cytokine IL-10 and T cell cytokine profile in periodontitis granulation tissue. Clin Exp Immunol. 2001;123:294-300.

29. Brem H, Golinko MS, Stojadinovic O, Kodra A, Diegelmann RF, Vukelic S, et al. Primary cultured fibroblasts derived from patients with chronic wounds: a methodology to produce human cell lines and test putative growth factor therapy such as GMCSF. J Transl Med. 2008;6:75-84.

30. Darby IA, Hewitson TD. Hypoxia in tissue repair and fibrosis. Cell Tissue Res. 2016;365:553-62.

31. Zafar F, Hinton RB, Moore RA, Baker RS, Bryant R, Narmoneva DA, et al. Physiological growth, remodeling potential, and preserved function of a novel bioprosthetic tricuspid valve: tubular bioprosthesis made of small intestinal submucosa-derived extracellular matrix. J Am Coll Cardiol. 2015;66:877-88.

32. Gerdisch MW, Shea RJ, Barron MD. Clinical experience with CorMatrix extracellular matrix in the surgical treatment of mitral valve disease. J Thorac Cardiovasc Surg. 2014;148:1370-8.

33. Woo JS, Fishbein MC, Reemtsen B. Histologic examination of decellularized porcine intestinal submucosa extracellular matrix (CorMatrix) in pediatric congenital heart surgery. Cardiovasc Pathol. 2016;25:12-7.

34. Padalino MA, Quarti A, Angeli E, Frigo AC, Vida VL, Pozzi M, et al. Early and mid-term clinical experience with extracellular matrix scaffold for congenital cardiac and vascular reconstructive surgery: a multicentric Italian study. Interact Cardiovasc Thorac Surg. 2015;21:40-9.

Key Words: trachea, trachea graft, trachea reconstruction, large animal model, small intestine submucosa, extracellular matrix, 3D-printing, polycaprolactone, tissue engineering 